\title{
Brain-lesion-induced hyperexploration
}

\author{
CHARLES M. MIEZEJESKI \\ New York State Institute for Basic Research in Mental Retardation, Staten Island, New York 10314
}

and

\author{
LEONARD W. HAMILTON \\ Rutgers University, New Brunswick, New Jersey 08903
}

\begin{abstract}
Adult male albino rats, seven with medial tegmental lesions, eight operated, and eight unoperated controls, were matched for preoperative levels of rearing behavior and then reexamined first at Postoperative Days 3, 7, 10, and 13 and again at Postoperative Days 25, 28,31, and 34. The frequency of rearing by the control groups exhibited a progressive postoperative decline. The rearing frequency of the lesioned rats steadily increased, reaching an asymptotic level well above that of the controls. Withinsession changes in rearing frequency and average response duration were also altered by the lesion. During each session the rearing of control rats decreased in frequency and increased in duration, while the responses of lesioned rats were frequent and of short duration throughout the session. These data indicate that medial tegmental lesions produce an increased frequency of brief exploratory rearing responses, supporting the notion of a medial tegmental syndrome of hyperreactivity.
\end{abstract}

The early lesion and stimulation experiments that demonstrated the role of the medial tegmentum in the control of the sleep-waking cycle (Jouvet, 1968; Kostowski, Giacalone, Garattini, \& Valzelli, 1968, 1969) have led to more specific examinations of this area's control of activity and reactivity. The effect of medial tegmental lesions on open-field behavior (Srebro \& Lorens, 1975), wheel running (Lorens, Sorensen, \& Yunger, 1971; Srebro \& Lorens, 1975), tilt-cage responding (Jacobs, Wise, \& Taylor, 1974), etc., has been explored. These studies revealed a lesion-induced increase in responsivity to exteroceptive stimulation. One way to maximize the information gained from studying the effect of a lesion on an animal's reactivity is to use a measure that is related to the organism's adaptive capacity. One such measure is the exploratory rearing that small mammals will emit when placed in a novel environment, which was noted in 1872 by Darwin (1965) and then reexamined in recent years by Lat (1965). Srebro and Lorens (1975) reported data suggesting that medial tegmental lesions may alter this behavior, but failed to provide conclusive evidence. With different testing conditions and more repetitions of the test, we obtained clear differences in rearing between lesioned and control rats.

\section{METHOD}

\section{Animals}

The animals were 26 male Sprague-Dawley rats (Blue Spruce Farms, Inc., Altamont, New York), 85-90 days of age and weighing $365-415 \mathrm{~g}$ at the time of surgery. They were experimentally naive and were housed in individual cages with ad-lib food and water. A 14/10 light/dark cycle, lights out at $2000 \mathrm{~h}$, was used.

Portions of this paper were presented at the annual meeting of the American Association for the Advancement of Science, February 1977. The authors thank George Collier for providing the apparatus used for recording rearing responses. Send reprint requests to Charles M. Miezejeski, Department of Neurobiology, New York State Institute for Basic Research in Mental Retardation, 1050 Forest Hill Road, Staten Island, New York 10314.

\section{Surgery and Histology}

All animals were anesthetized by IP injection of $.3 \mathrm{cc} / 100 \mathrm{~g}$ of body weight of Equi-Thesin (Jensen-Salisbury) and positioned in the stereotaxic instrument with the incisor bar at $-2.4 \mathrm{~mm}$. A stainless steel electrode, insulated with Epoxylite, except for $.5 \mathrm{~mm}$ at the tip, was used. The midsaggital sinus was pushed aside with the tip of the electrode by using the lateral adjustment of the stereotaxic instrument. The electrode was then positioned on the midline $.7 \mathrm{~mm}$ posterior to lambda and was lowered to $6.5 \mathrm{~mm}$ and $4.8 \mathrm{~mm}$ beneath the dura. At each point, a lesion was made by passing $1.5 \mathrm{~mA}$ of anodal current for $10 \mathrm{sec}$ with the cathode clipped to the edge of the scalp wound. Operated controls were treated similarly except for the lowering of the electrode and the passage of the current. Ten animals received lesions, eight rats were operated controls, and eight normal controls received IP injections of Equi-Thesin but did not undergo surgery. This normal control group was included in the experiment to control for the possible effects of damage to the operated controls and lesioned animals during the manipulation of the midsaggital sinus, as described above. It was thought that either ischemia or direct damage from this manipulation might produce an increase in rearing behavior comparable to that observed by Nakajima (1964) following injections of potassium chloride into the superior colliculus of rats.

Following completion of behavioral testing, the animals were sacrificed with an overdose of Equi-Thesin and were perfused intracardially with saline followed by a $10 \%$ Formalin saline solution. The brains were embedded in paraffin and 10-micron representative sections were taken every 200 microns and stained with cresyl violet.

\section{Apparatus}

The apparatus used for the automatic recording of the frequency and duration of rearing responses was the same as that described in Miezejeski, Lamon, Collier, and Hamilton (1976), except that the photobeams (each covered with a red Wrattan gel filter) were $12 \mathrm{~cm}$ above the floor and a bank of five counters and five timers was used to record the frequency and total duration of rearing during each of five 2-min intervals.

\section{Procedure}

All animals were handled 6 days before and again 4 days before their first exposure to the rearing apparatus. Rearing activity during a 10-min exposure was recorded on 10 occasions: 6 and 3 days preoperatively, at 3-day intervals between Post- 
operative Days 4 and 13 (Block A), and at 3-day intervals between Postoperative Days 25 and 34 (Block B). All animals were tested between 0700 and $1200 \mathrm{~h}$ and in a random sequence to prevent a correspondence between group and time of test. Body weight was recorded at the time of surgery and after each postoperative test.

\section{Statistical Analyses}

Analyses of variance for three factors (groups, days, and 2-min intervals) with repeated measures on two factors (days and 2-min intervals) were performed on the frequency of rearing and average duration data, both preoperative and postoperative. Analyses of variance for one factor (groups), preoperative data, and two factors (groups and days), postoperative data, with repeated measures on one factor (days) were performed on the body weight data. Further comparisons of differences revealed by the overall ANOVAs were made using Fisher's 1.s.d. test (Federer, 1955) with an alpha level of .05. Applications of the 1.s.d. test to differences revealed by significant interactions used a hybrid error term which represented the addition of the respective error terms for the plot and subplot variables (Winer, 1971). It was felt that this provided a more conservative application of the 1.s.d. test for the ANOVA technique used.

\section{RESULTS}

\section{Histology}

Figure 1 depicts representative sections of the smallest (darkened area) and largest (outlined area) acceptable lesions. Although the dorsal raphe nucleus was the only cell group consistently destroyed in all seven rats with acceptable lesions, there was also complete or partial destruction of the median raphe nucleus $(n=6)$, the ventral tegmental nuclei $(n=5)$, the medial longitudinal fasciculus $(n=5)$, and the decussation of the brachium conjunctivum $(n=4)$ (nomenclature according to Pellegrino and Cushman, 1967). Of the remaining three rats receiving lesions, one died before the completion of testing and two had lesions that failed to meet the histological criteria.

\section{Rearing}

Although the groups were matched for preoperative level of rearing, ANOVAs were performed to determine if any difference existed between the groups due to the subsequent loss of animals in the lesioned group. The analyses yielded neither a significant groups effect nor a significant interaction with groups.

As can be seen in Figure 2, during both Blocks A and $B$ the lesioned rats reared more often than either of the control groups $[F(2,20)=7.51$ and 25.56 , respectively, $\mathrm{p}<.01]$. Only during Block $\mathrm{A}$ was there an interaction of Groups by Days $[F(6,60)=4.46, p<.01]$. Fisher's 1.s.d. test yielded a critical value of 8.2 , indicating that the rearing frequency of the lesioned rats increased significantly with each successive test during Block A but decreased from the beginning to the end of Block A in both the normal and operated control groups; however, the peak response frequency of the operated controls was on the second test of Block A (Postoperative Day 7), while that of the normal controls was on the first test of Block A (Postoperative Day 4). The difference between the operated and normal controls is

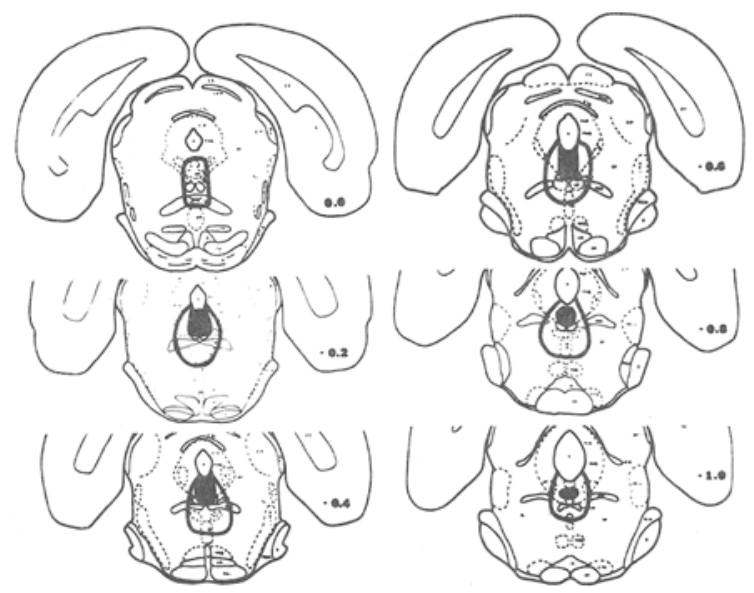

Figure 1. Coronal sections displaying the minimum (darkened area) and maximum (solid outline) acceptable extents of damage. The A-P location is indicated in the lower right area of each section. See Pellegrino and Cushman (1967) for abbreviations.

further evidenced by a significant difference between the two groups on Postoperative Day 4. The initial decline in the rearing of the operated controls may be symptomatic of a general postoperative malaise, since the response frequency of these rats was equal to that of the normal controls on subsequent test days. The lesioned animals reared more frequently than the operated controls on all postoperative days and more frequently than the normal controls on all but Postoperative Day 4.

As Figure 3 indicates, there was an effect of 2-min intervals in both Blocks A and $B[F(4,80)=9.63$ and 7.07, respectively, $\mathrm{p}<.01$ ] and a Groups by 2-Min Intervals interaction $[F(8,80)=2.37$ and $9.49, p<.05$ and $<.01$, respectively]. Fisher's 1.s.d. test yielded a critical value of 3.53 for Block $A$, indicating that the lesioned animals reared more frequently than either the operated or normal controls during all 2-min intervals of Block A except the first. The rearing frequency of the lesioned rats maintained a high and unchanging level across each session of Block $A$, indicating a lack of habituation; the response frequency of the normal

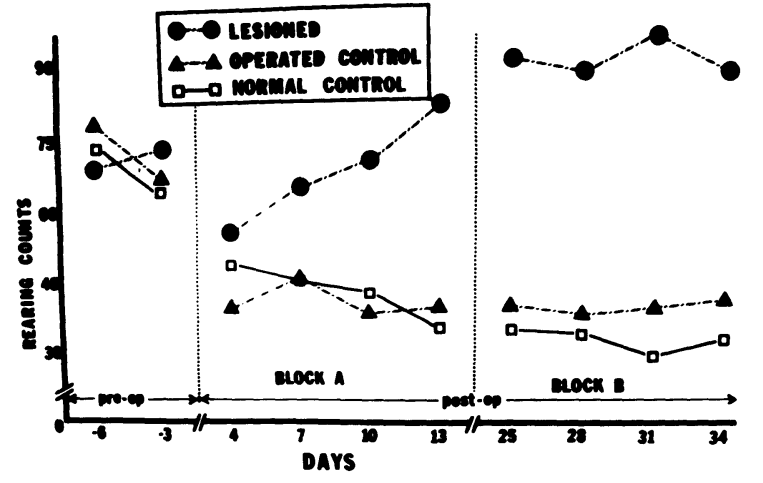

Figure 2. Mean frequency of rearing on 10-min tests given twice preoperatively, four times during an early (Block A) and four times during a later (Block B) postoperative period. 

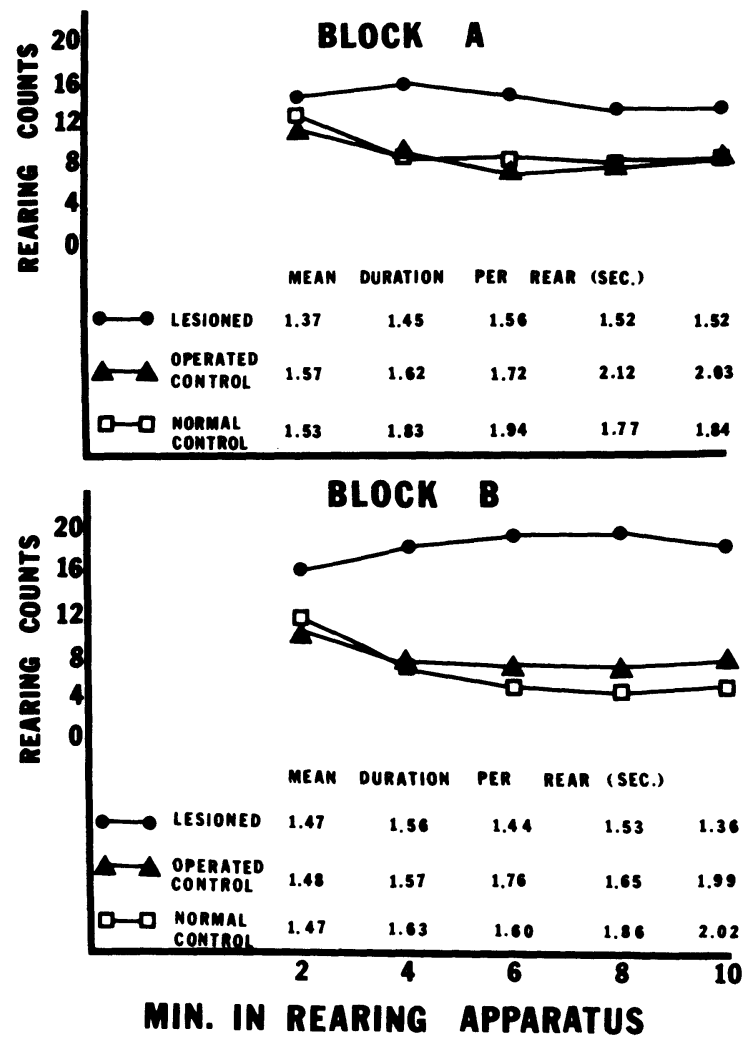

Figure 3. Mean frequency of rearing during each of five successive 2-min intervals collapsed across days. Upper graph: Postoperative Days 4, 7, 10, and 13; lower graph: Postoperative Days $25,28,31$, and 34 . The tabular insets provide the corresponding mean duration per rear data.

controls was lower during the last four 2-min intervals than during the first 2-min interval and that of the operated controls decreased between the first and third 2-min intervals. The critical value for the data of Block B was 3.87, showing a clear separation of the frequency scores for the lesioned group from those of both control groups during all 2-min intervals. The normal controls exhibited the same pattern of declining response frequency across each session of Block B as they had for Block A. On the other hand, both the lesioned rats and the operated controls showed no significant withinsession changes, indicating a lack of habituation.

During some 2-min intervals in both Blocks A and B, a few animals in both the operated and normal control groups emitted no rearing responses at all, leaving empty cells in the ANOVAs for average response duration. Replacement scores were obtained for the empty cells by using the mean of the combination of the other scores obtained on that postoperative day during the other 2-min intervals and the scores obtained during that 2-min interval on other postoperative days (Winer, 1971). During Block A, one rat in the operated control group had neither emitted any responses during any 2-min interval on Postoperative Day 7 nor any responses during the fourth 2-min interval on any of the postoperative days, making it impossible to calculate replacement scores for any of these cells. Thus, that animal's data was eliminated from the analysis. The ANOVAs for average response duration indicate, as do the tabular insets of Figure 3, that rearing duration increased during the 10 -min sessions $[F(4,69)=2.76, p<.05$ for Block $A$ and $F(4,67)=3.84, p<.01$ for Block B]. The ANOVA for the Block B duration data revealed a Groups by 2-Min Intervals interaction $[F(8,67)=2.33, p<.05]$ with a critical value of .37 (1.s.d. test), indicating that, while the average duration of the rearing response did not change for the lesioned animals, it increased from the beginning to the end of each session for both control groups. The average duration of the control rats' rearing responses during the last 2-min interval was significantly greater than that of the lesioned rats. Thus, the lesioned rats emitted more frequent, but briefer, rearing responses during the last few minutes of each session.

\section{Body Weight}

The ANOVA of the preoperative body weights indicated no differences between the three groups prior to surgery $(F<1)$. Analysis of postoperative body weights revealed a groups difference $[F(2,20)=4.80, p<.05]$, a change across days $[F(7,140)=368.3, p<.01]$, and no Groups by Days interaction. A follow-up analysis yielded a critical value of $27.8 \mathrm{~g}$ (1.s.d. test), indicating that the groups effect was due to the difference between the lesioned group (mean postoperative body weight $=$ $408.6 \mathrm{~g}$ ) and the normal controls (mean postoperative body weight $=449.6 \mathrm{~g}$ ). The operated control group (mean postoperative body weight $=425.3 \mathrm{~g}$ ) did not differ from either of the other two groups.

\section{DISCUSSION}

There is little doubt that the medial tegmental lesion had an effect on rearing behavior as measured here. Why then did not Srebro and Lorens (1975) observe such an effect? Two conditions appear to be of particular importance: (1) level of test chamber illumination and (2) number of tests. In our experiment, there was a total of 10 testing sessions, all conducted in the dark; in their study, the responses were observed only during two postoperative tests, using an illuminated open field with a white floor. Rearing is highly sensitive to testing conditions and is a rapidly habituating response. When placed in a brightly illuminated open field, a rat exhibits a pattern of rearing characterized by an initial depression, a subsequent increase to a peak level, and then habituation throughout the remainder of the test (Lat, 1965). This is precisely the pattern exhibited by the control group in the Srebro and Lorens (1975) study. Moreover, in their experiment, the lesioned group most comparable to the lesioned group in our experiment displayed a pattern of increasing rearing across both test sessions (Srebro \& Lorens, 1975, Figure 6). Thus, by testing their rats in an illuminated open field and consequently dampening the overall level of rearing, Srebro and Lorens decreased the likelihood of observing lesion-induced differences. Second, the significant effect observed in our experiment may have been somewhat influenced by the use of repeated pre- and postoperative testing. On the 10th test of our experiment, the operated and normal controls were responding at a level that was $53 \%$ and $44 \%$, respectively, of that observed on the first preoperative test. Although the medial tegmental lesion may have caused the rearing behavior of the lesioned rats to habituate more slowly than that of the controls, an explanation based solely on a lesion-induced change in habituation is clearly inadequate. The average frequency of rearing by the 
lesioned rats on the last test day was $129 \%$ of that on the first preoperative test, and one rat in this group was responding at $235 \%$ of its initial level.

The above mention of habituation refers to this process in terms of a decline across days. Habituation research, however, has been more traditionally concerned with within-session changes. Although the present experiment was not designed for the purpose of examining within-session habituation, the observed within-session changes in rearing are not entirely what one might have expected, although neither are they exceptional. During Block A, the operated controls exhibited essentially the same within-session pattern of declining response frequency as did the normal controls; during Block B, they failed to do so and, in this respect, were more like the lesioned animals. In spite of this, as Figure 3 reveals, during both Blocks A and B both control groups reared markedly less frequently than the lesioned animals and differed very little overall. The similarity of these two control groups is further accentuated by the occurrence of comparable increases in average response duration from the beginning to the end of each test session. Clearly, the control operation did not increase rearing activity, and its apparent effect on within-session habituation is far from extraordinary and may be due to nonspecific effects.

One of the lesioned rats in our experiment suffered damage that was limited to the dorsal raphe nucleus and a small portion of the surrounding central gray substance. This rat, nonetheless, exhibited a postoperative level of rearing well above that of any control animal. In a subsequent experiment, reported elsewhere, we demonstrated that the dorsomedial tegmental lesion and perhaps damage specific to the dorsal raphe nucleus is, in fact, responsible for the increased rearing reported here (Miezejeski, Note 1). Thus, recent research, including the present results, observations of increased tilt-cage responding (Miezejeski, 1977), and elevated shock-induced aggression (Jacobs \& Cohen, 1976) support the hypothesis that dorsomedial tegmental lesions produce increased reactivity to exteroceptive stimulation.

In a very recent report, lesions of the pretectum were shown to increase the total duration of rearing in rats (Weldon \& Smith, Note 2). The apparently similar effects following lesions of either the dorsomedial tegmentum or the pretectum suggest a functional relationship between these areas. The relationship is supported further by neuroanatomical experiments demonstrating connections between the midbrain raphe nuclei and the pretectum (Fuxe, 1965). The similar effect on rearing of dorsomedial tegmental or pretectal damage tends to emphasize the role of the visual system in this process. While an altered reactivity to or altered perception of visual stimuli may contribute to the increased rearing produced by lesions of either of these regions, the nature of the hyperreactivity syndrome produced by medial tegmental lesions does not appear to be restricted to this modality. Combined lesions of the dorsomedial and ventromedial tegmentum have also been shown to enhance the auditory startle reaction of rats (Davis \& Sheard, 1974) and reactivity to gustatory stimuli (Miezejeski, 1977). In conclusion, the results reported here provide additional support for the notion that medial tegmental lesions produce a syndrome of hyperreactivity.

\section{REFERENCE NOTES}

1. Miezejeski, C. M. Hypoactive, hyperreactive rats following limbic midbrain lesions. Paper presented at the annual meeting of the Eastern Psychological Association, Boston, April 1977.

2. Weldon, D. A., \& Smith, C. J. Activity and rearing following lesions of superior colliculus, posterior thalamus, or both: Nonadditive effects. Paper presented at the annual meeting of the Eastern Psychological Association, Boston, April 1977.

\section{REFERENCES}

DARWIN, C. The expression of the emotions in man and animals. Chicago: University of Chicago Press, 1965.

Davis, M., \& Sheard, M. H. Habituation and sensitization of the rat startle response: Effects of raphe lesions. Physiology and Behavior, 1974, 12, 425-431.

Federer, W. T. Experimental design. New York: Macmillan, 1955.

FuxE, K. Evidence for the existence of monoamine neurons in the central nervous system: IV. Distribution of monoamine nerve terminals in the central nervous system. Acta Physiologica Scandinavica, 1965, 64, 41-85 (Suppl. 247).

JACoBS, B. L., \& Cohen, A. Differential behavioral effects of lesions of the median or dorsal raphe nuclei in rats: Open field and pain-elicited aggression. Journal of Comparative and Physiological Psychology, 1976, 90, $102-108$.

JACOBS, B. L., WISE, W. D., \& TAYloR, K. M. Differential behavioral and neurochemical effects following lesions of the dorsal or median raphe nuclei in rats. Brain Research, 1974, 79, 353-361.

Jouvet, M. Insomnia and decrease of cerebral - 5hydroxytryptamine after destruction of the raphe system in the cat. In S. Garattini \& P. A. Shore (Eds.), Advances in pharmacology (Vol.6B). New York: Academic Press, 1968.

Kostowski, W., Giacalone, E., Garattini, S., \& VAlzelli, L. Studies on behavioral and biochemical changes in rats after lesion of midbrain raphe. European Journal of Pharmacology, 1968, 4, 371-376.

Kostowski, W., Giacalone, E., Garattini, S., \& VALzELLI, L. Electrical stimulation of midbrain raphe: Biochemical, behavioral and bioelectrical effects. European Journal of Pharmacology, 1969, 7, 170-175.

LAT, J. The spontaneous exploratory reactions as a tool for psychopharmacological studies: A contribution towards a "theory" of contradictory results in psychopharmacology. In M. Ya. Mikhel'son \& V. G. Longo (Eds.), Pharmacology of conditioning, learning and retention: Proceedings of the 2nd international meeting, Prague (Vol. 1). Oxford: Pergamon, 1965.

Lorens, S. A., Sorenson, J. P., \& Yunger, L. M. Behavioral and neurochemical effects of lesions in the raphe system of the rat. Journal of Comparative and Physiological Psychology, 1971, 77, 48-52.

Miezejeski, C. M. Persistent hyperresponsivity following medial tegmental lesions: Exteroceptive versus interoceptive stimulation. Life Sciences, 1977, 20, 2087-2092.

Miezejeski, C. M., Lamon, S., Collier, G., \& Hamilton, L. W. Partitioning of behavior arousal. Physiology and Behavior, 1976, 17, 581-586.

NaKajima, S. Effects of chemical injection into the reticular formation of rats. Journal of Comparative and Physiological Psychology, 1964, 58, 10-15.

Pellegrino, L. J., \& Cushman, A. J. A stereotaxic atlas of the rat brain. New York: Appleton-Century-Crofts, 1967.

SRebro, B., \& Lorens, S. A. Behavioral effects of selective midbrain raphe lesions in the rat. Brain Research, 1975, 89, 303-325.

WINER, B. J. Statistical principles in experimental design. New York: McGraw-Hill, 1971.

(Received for publication June 22, 1977.) 